\title{
POLSAR LAND COVER CLASSIFICATION BASED ON HIDDEN POLARIMETRIC FEATURES IN ROTATION DOMAIN AND SVM CLASSIFIER
}

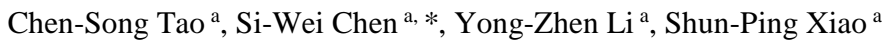 \\ a The State Key Laboratory of Complex Electromagnetic Environment Effects on Electronics and Information System, School of \\ Electronics Science and Engineering, National University of Defense Technology, Changsha, 410073, China - \\ chenswnudt@163.com
}

Commission I, WG I/3

KEY WORDS: Polarimetric Synthetic Aperture Radar (PolSAR), Polarimetric Feature Mining, Rotation Domain, Support Vector Machine (SVM), Land Cover Classification

\begin{abstract}
:
Land cover classification is an important application for polarimetric synthetic aperture radar (PolSAR) data utilization. Rollinvariant polarimetric features such as $H / A n i / \bar{\alpha} /$ Span are commonly adopted in PolSAR land cover classification. However, target orientation diversity effect makes PolSAR images understanding and interpretation difficult. Only using the roll-invariant polarimetric features may introduce ambiguity in the interpretation of targets' scattering mechanisms and limit the followed classification accuracy. To address this problem, this work firstly focuses on hidden polarimetric feature mining in the rotation domain along the radar line of sight using the recently reported uniform polarimetric matrix rotation theory and the visualization and characterization tool of polarimetric coherence pattern. The former rotates the acquired polarimetric matrix along the radar line of sight and fully describes the rotation characteristics of each entry of the matrix. Sets of new polarimetric features are derived to describe the hidden scattering information of the target in the rotation domain. The latter extends the traditional polarimetric coherence at a given rotation angle to the rotation domain for complete interpretation. A visualization and characterization tool is established to derive new polarimetric features for hidden information exploration. Then, a classification scheme is developed combing both the selected new hidden polarimetric features in rotation domain and the commonly used roll-invariant polarimetric features with a support vector machine (SVM) classifier. Comparison experiments based on AIRSAR and multi-temporal UAVSAR data demonstrate that compared with the conventional classification scheme which only uses the roll-invariant polarimetric features, the proposed classification scheme achieves both higher classification accuracy and better robustness. For AIRSAR data, the overall classification accuracy with the proposed classification scheme is $94.91 \%$, while that with the conventional classification scheme is 93.70\%. Moreover, for multi-temporal UAVSAR data, the averaged overall classification accuracy with the proposed classification scheme is up to $97.08 \%$, which is much higher than the $87.79 \%$ from the conventional classification scheme. Furthermore, for multitemporal PolSAR data, the proposed classification scheme can achieve better robustness. The comparison studies also clearly demonstrate that mining and utilization of hidden polarimetric features and information in the rotation domain can gain the added benefits for PolSAR land cover classification and provide a new vision for PolSAR image interpretation and application.
\end{abstract}

\section{INTRODUCTION}

With the ability to work day and night, under all weather conditions, polarimetric synthetic aperture radar (PolSAR), which can acquire full polarization information of targets by transmitting and receiving microwaves with specific polarization states has become one of the most important and promising remote sensors (Lee and Pottier 2009). Amounts of successful applications have been developed with increasing PolSAR data (Lee and Pottier 2009; Chen and Sato 2013; Chen et al. 2016a). As a common key step for environment monitoring, general survey of crop and appraisal of cultivated and urban land occupation, land cover classification is an important application direction for understanding and interpreting PolSAR images. The classification result with high accuracy can provide information support to these application fields (Wu et al. 2007).

Generally, there are two kinds of approaches to improve the accuracy of PolSAR land cover classification. The first kind of approaches focus on mining and selecting polarimetric features. Through modeling and interpreting the polarimetric scattering mechanism finely (Cloude and Pottier 1996; Freeman and Durden 1998; Yamaguchi et al. 2005; Chen et al. 2014a; Chen et al. 2014b), these approaches extract the polarimetric features with better discriminate among different land covers from the acquired full polarization information. Moreover, the second kind of approaches to improve the classification accuracy try to find out the classifier with the better classification performance to take full advantage of the available polarimetric features (Cloude and Pottier 1997; Lee et al. 1999; Zhou et al. 2016). Certainly, selecting polarimetric feature and classifier at the same time is also an effective way to improve the accuracy of PolSAR land cover classification.

Roll-invariant polarimetric features are commonly adopted in the classification schemes based on polarimetric features. There is a conventional classification scheme only using the entropy $H$, anisotropy $A n i$, mean alpha angle $\bar{\alpha}$, and total scattering power Span (Cloude and Pottier 1997). However, polarimetric response of a target is strongly dependent on its orientation (Chen et al. 2014c). The backscattering responses of the same target with different orientations are significantly various. On the other hand, the backscattering responses of different targets with some specific orientations may be quite similar with each other. For example, with the orientation diversity, buildings in urban areas and vegetation in forest areas are the difficult points in PolSAR image understanding (Chen et al. 2014c). This frequently introduces ambiguity in the interpretation of scattering mechanisms and limits the accuracy of the

\footnotetext{
* Corresponding author
} 
conventional classification scheme, which only uses the rollinvariant polarimetric features of $H / A n i / \bar{\alpha} /$ Span .

To address this problem, certain exploration of targets' orientation diversity is made and two novel methods, which are named uniform polarimetric matrix rotation theory (Chen et al. 2014c) and a visualization and characterization tool of polarimetric coherence pattern (Chen et al. 2016b; Chen 2017) respectively were proposed to mine and extract the hidden polarimetric features in the rotation domain along the radar line of sight. The former rotates the acquired polarimetric matrix (a coherency matrix or covariance matrix usually) along the radar line of sight and fully describes the rotation characteristics of each entry of the matrix. Series of new polarimetric features are derived to describe the hidden scattering information of the target in the rotation domain (Chen et al. 2014c). The latter extends the traditional polarimetric coherence at a given rotation angle to the whole rotation domain in order to explore the complete interpretation of targets' polarimetric coherence. A visualization and characterization tool which covers all rotation angles along the radar line of sight is investigated and established to derive other new polarimetric features for hidden information exploration (Chen et al. 2016b; Chen 2017). The polarimetric features derived from the uniform polarimetric matrix rotation theory and the visualization and characterization tool of polarimetric coherence pattern are both the hidden polarimetric features mined in the rotation domain and parts of them were applied to crop discrimination (Chen et al. 2015), target enhancement (Chen et al. 2014c) and manmade target extraction (Xiao et al. 2014) successfully.

Because these new polarimetric features contain some hidden scattering information of targets in the rotation domain and relate to their orientations directly. This work applies them to PolSAR land cover classification investigation. In this work, we select suitable hidden polarimetric features derived from the rotation domain according to the criterion of the class separation distance, combine the selected hidden polarimetric features with the commonly used roll-invariant polarimetric features of $H / A n i / \bar{\alpha} /$ Span to achieve better classification performance by complementing the land cover discrimination abilities of both, and develop a classification scheme using the combination of the polarimetric features as input for the support vector machine (SVM) (Chang and Lin 2011) classifier with relatively good classification performance. Because of the added benefits from hidden polarimetric feature mining in the rotation domain, the proposed classification scheme is able to achieve both higher classification accuracy and better robustness than those of the conventional classification scheme which only uses the roll-invariant polarimetric features of $H / A n i / \bar{\alpha} /$ Span

\section{HIDDEN POLARIMETRIC FEATURE MINING IN THE ROTATION DOMAIN}

\subsection{Polarimetric Matrixes and Their Rotation}

For PolSAR, in the horizontal and vertical polarization basis $(H, V)$, the acquired full polarization information can form a scattering matrix with the representation as

$$
\mathbf{S}=\left[\begin{array}{ll}
S_{H H} & S_{H V} \\
S_{V H} & S_{V V}
\end{array}\right]
$$

where $S_{V H}$ is the backscattered coefficient from horizontal transmitting and vertical receiving polarization. Other terms are defined similarly.
Subject to the reciprocity condition $\left(S_{H V}=S_{V H}\right)$, the coherency matrix is

$$
\mathbf{T}=\left\langle\mathbf{k}_{P} \mathbf{k}_{P}^{H}\right\rangle=\left[\begin{array}{lll}
T_{11} & T_{12} & T_{13} \\
T_{21} & T_{22} & T_{23} \\
T_{31} & T_{32} & T_{33}
\end{array}\right]
$$

where $\mathbf{k}_{P}=(1 / \sqrt{2})\left[\begin{array}{lll}S_{H H}+S_{V V} & S_{H H}-S_{V V} & 2 S_{H V}\end{array}\right]^{T}$ is the Pauli scattering vector, $\langle\cdot\rangle$ denotes the sample average, the superscript ${ }^{T}$ and ${ }^{H}$ denote the transpose and conjugate transpose respectively, and $T_{i j}$ is the $(i, j)$ entry of the coherency matrix $\mathbf{T}$.

With a rotation angle $\theta$ along the radar line of sight, the rotated scattering matrix $\mathbf{S}(\theta)$ and coherency matrix $\mathbf{T}(\theta)$ respectively become

$$
\begin{gathered}
\mathbf{S}(\theta)=\mathbf{R}_{2}(\theta) \mathbf{S R}_{2}^{H}(\theta) \\
\mathbf{T}(\theta)=\mathbf{k}_{P}(\theta) \mathbf{k}_{P}^{H}(\theta)=\mathbf{R}_{3}(\theta) \mathbf{T R}_{3}^{H}(\theta)
\end{gathered}
$$

where the rotation matrixes are $\mathbf{R}_{2}(\theta)=\left[\begin{array}{cc}\cos \theta & \sin \theta \\ -\sin \theta & \cos \theta\end{array}\right]$ and $\mathbf{R}_{3}(\theta)=\left[\begin{array}{ccc}1 & 0 & 0 \\ 0 & \cos 2 \theta & \sin 2 \theta \\ 0 & -\sin 2 \theta & \cos 2 \theta\end{array}\right]$.

\subsection{Uniform Polarimetric Matrix Rotation Theory}

Based on (4), the elements of $\mathbf{T}(\theta)$ are

$T_{11}(\theta)=T_{11}$

$T_{12}(\theta)=T_{12} \cos 2 \theta+T_{13} \sin 2 \theta$

$T_{13}(\theta)=-T_{12} \sin 2 \theta+T_{13} \cos 2 \theta$

$T_{23}(\theta)=(1 / 2)\left(T_{33}-T_{22}\right) \sin 4 \theta+\operatorname{Re}\left[T_{23}\right] \cos 4 \theta+j \operatorname{Im}\left[T_{23}\right]$

$T_{22}(\theta)=T_{22} \cos ^{2} 2 \theta+T_{33} \sin ^{2} 2 \theta+\operatorname{Re}\left[T_{23}\right] \sin 4 \theta$

$T_{33}(\theta)=T_{22} \sin ^{2} 2 \theta+T_{33} \cos ^{2} 2 \theta-\operatorname{Re}\left[T_{23}\right] \sin 4 \theta$

and the powers of the off-diagonal terms of $\mathbf{T}(\theta)$ are also taken into consideration

$\left|T_{12}(\theta)\right|^{2}=\left|T_{12}\right|^{2} \cos ^{2} 2 \theta+\left|T_{13}\right|^{2} \sin ^{2} 2 \theta+\operatorname{Re}\left[T_{12} T_{13}^{*}\right] \sin 4 \theta$

$\left|T_{13}(\theta)\right|^{2}=\left|T_{12}\right|^{2} \sin ^{2} 2 \theta+\left|T_{13}\right|^{2} \cos ^{2} 2 \theta-\operatorname{Re}\left[T_{12} T_{13}^{*}\right] \sin 4 \theta$

$$
\begin{aligned}
\left|T_{23}(\theta)\right|^{2}= & \frac{1}{4}\left(T_{33}-T_{22}\right)^{2} \sin ^{2} 4 \theta+\operatorname{Re}^{2}\left[T_{23}\right] \cos ^{2} 4 \theta \\
& +\frac{1}{2}\left(T_{33}-T_{22}\right) \operatorname{Re}\left[T_{23}\right] \sin 8 \theta+\operatorname{Im}^{2}\left[T_{23}\right]
\end{aligned}
$$

where $\operatorname{Re}\left[T_{i j}\right]$ and $\operatorname{Im}\left[T_{i j}\right]$ are the real and imaginary parts of $T_{i j}$ respectively.

With simple mathematic transformations for (5)-(13), all the elements and the powers of the off-diagonal terms of a rotated coherency matrix $\mathbf{T}(\theta)$ can be represented as a uniform sinusoidal function

$$
f(\theta)=A \sin \left[\omega\left(\theta+\theta_{0}\right)\right]+B
$$


where $A$ is the oscillation amplitude, $B$ is the oscillation center, $\omega$ is the angular frequency and $\theta_{0}$ is the initial angle.

Therefore, the new polarimetric feature parameter set $\left\{A, B, \omega, \theta_{0}\right\}$ named as oscillation parameter set is able to completely characterize the rotation properties of all the elements and the powers of the off-diagonal terms of a coherency matrix rotated along the radar line of sight. That is the uniform polarimetric matrix rotation theory (Chen et al. 2014c). And the hidden polarimetric features derived from it are summarized in Table 1 (Chen et al. 2014c).

\begin{tabular}{|c|c|c|c|c|}
\hline & $A=\sqrt{\cdot}$ & $B$ & $\omega$ & $\theta_{0}=\frac{1}{\omega}$ Angle \{\} \\
\hline $\operatorname{Re}\left[T_{12}(\theta)\right]$ & $\operatorname{Re}^{2}\left[T_{12}\right]+\operatorname{Re}^{2}\left[T_{13}\right]$ & 0 & 2 & $\operatorname{Re}\left[T_{13}\right]+j \operatorname{Re}\left[T_{12}\right]$ \\
\hline $\operatorname{Re}\left[T_{13}(\theta)\right]$ & $\operatorname{Re}^{2}\left[T_{12}\right]+\operatorname{Re}^{2}\left[T_{13}\right]$ & 0 & 2 & $-\operatorname{Re}\left[T_{12}\right]+j \operatorname{Re}\left[T_{13}\right]$ \\
\hline $\operatorname{Im}\left[T_{12}(\theta)\right]$ & $\operatorname{Im}^{2}\left[T_{12}\right]+\operatorname{Im}^{2}\left[T_{13}\right]$ & 0 & 2 & $\operatorname{Im}\left[T_{13}\right]+j \operatorname{Im}\left[T_{12}\right]$ \\
\hline $\operatorname{Im}\left[T_{13}(\theta)\right]$ & $\operatorname{Im}^{2}\left[T_{12}\right]+\operatorname{Im}^{2}\left[T_{13}\right]$ & 0 & 2 & $-\operatorname{Im}\left[T_{12}\right]+j \operatorname{Im}\left[T_{13}\right]$ \\
\hline $\operatorname{Re}\left[T_{23}(\theta)\right]$ & $\frac{1}{4}\left(T_{33}-T_{22}\right)^{2}+\operatorname{Re}^{2}\left[T_{23}\right]$ & 0 & 4 & $\frac{1}{2}\left(T_{33}-T_{22}\right)+j \operatorname{Re}\left[T_{23}\right]$ \\
\hline$T_{22}(\theta)$ & $\frac{1}{4}\left(T_{33}-T_{22}\right)^{2}+\operatorname{Re}^{2}\left[T_{23}\right]$ & $\frac{1}{2}\left(T_{22}+T_{33}\right)$ & 4 & $\operatorname{Re}\left[T_{23}\right]+j \frac{1}{2}\left(T_{22}-T_{33}\right)$ \\
\hline$T_{33}(\theta)$ & $\frac{1}{4}\left(T_{33}-T_{22}\right)^{2}+\operatorname{Re}^{2}\left[T_{23}\right]$ & $\frac{1}{2}\left(T_{22}+T_{33}\right)$ & 4 & $-\operatorname{Re}\left[T_{23}\right]+j \frac{1}{2}\left(T_{33}-T_{22}\right)$ \\
\hline$\left|T_{12}(\theta)\right|^{2}$ & $\operatorname{Re}^{2}\left[T_{12} T_{13}^{*}\right]+\frac{1}{4}\left(\left|T_{13}\right|^{2}-\left|T_{12}\right|^{2}\right)^{2}$ & $\frac{1}{2}\left(\left|T_{12}\right|^{2}+\left|T_{13}\right|^{2}\right)$ & 4 & $-\operatorname{Re}\left[T_{12} T_{13}^{*}\right]+j \frac{1}{2}\left(\left|T_{12}\right|^{2}-\left|T_{13}\right|^{2}\right)$ \\
\hline$\left|T_{13}(\theta)\right|^{2}$ & $\operatorname{Re}^{2}\left[T_{12} T_{13}^{*}\right]+\frac{1}{4}\left(\left|T_{13}\right|^{2}-\left|T_{12}\right|^{2}\right)^{2}$ & $\frac{1}{2}\left(\left|T_{12}\right|^{2}+\left|T_{13}\right|^{2}\right)$ & $\frac{1}{2}\left(\left|T_{13}\right|^{2}-\left|T_{12}\right|^{2}\right)$ \\
\hline$\left|T_{23}(\theta)\right|^{2}$ & $\frac{1}{4}\left\{\frac{1}{4}\left(T_{33}-T_{22}\right)^{2}+\operatorname{Re}^{2}\left[T_{23}\right]\right\}^{2}$ & $\frac{1}{2}\left\{\frac{1}{4}\left(T_{33}-T_{22}\right)^{2}+\operatorname{Re}^{2}\left[T_{23}\right]\right\}+\operatorname{Im}^{2}\left[T_{23}\right]$ & 8 & $\frac{1}{2}\left(T_{33}-T_{22}\right) \operatorname{Re}\left[T_{23}\right]+$ \\
\end{tabular}

Table 1 Polarimetric features derived from the uniform polarimetric matrix rotation theory

where the superscript ${ }^{*}$ denotes the conjugate, Angle $\{a\}$ is the phase of $a$ in the complex axis and the range of it is $[-\pi, \pi]$. There are eleven independent hidden polarimetric features in Table 1. They are five initial angle parameters $\left(\theta_{0}-\operatorname{Re}\left[T_{12}(\theta)\right], \theta_{0}-\operatorname{Im}\left[T_{12}(\theta)\right], \theta_{0} \operatorname{Re}\left[T_{23}(\theta)\right]\right.$, $\left.\theta_{0-}\left|T_{12}(\theta)\right|^{2}, \theta_{0-}\left|T_{23}(\theta)\right|^{2}\right)$, four oscillation amplitude parameters $\quad\left(A_{-} \operatorname{Re}\left[T_{12}(\theta)\right] \quad, \quad A_{-} \operatorname{Im}\left[T_{12}(\theta)\right]\right.$, $A_{-}\left|T_{12}(\theta)\right|^{2}, A_{-}\left|T_{23}(\theta)\right|^{2}$ ) and two oscillation center parameters $\left(B_{-} T_{22}(\theta), B_{-}\left|T_{23}(\theta)\right|^{2}\right)$.

\subsection{A Visualization and Characterization Tool of Polarimetric Coherence Pattern}

In theory, based on two polarization channels $s_{1}$ and $s_{2}$, the polarimetric coherence is defined as

$$
\left|\gamma_{1-2}\right|=\frac{\left|E\left(s_{1} s_{2}^{*}\right)\right|}{\sqrt{E\left(\left|s_{1}\right|^{2}\right) E\left(\left|s_{2}\right|^{2}\right)}}
$$

where $E(s)$ is the expectation of $s$, and the value of $\left|\gamma_{1-2}\right|$ is within the range of $[0,1]$.

However, in practice, the sample average of sufficient samples with similar properties is used to estimate the polarimetric coherence as

$$
\left|\gamma_{1-2}\right|=\frac{\left|\left\langle s_{1} s_{2}^{*}\right\rangle\right|}{\sqrt{\left\langle\left. s_{1}\right|^{2}\right\rangle\left\langle\left|s_{2}\right|^{2}\right\rangle}}
$$

In order to explore the complete interpretation of targets' polarimetric coherence, the original polarimetric coherence at a given rotation angle $(\theta=0)$ is extended to the whole rotation domain, which covers all rotation angles $(\theta \in[-\pi, \pi))$ along the radar line of sight. That is the polarimetric coherence pattern (Chen et al. 2016b; Chen 2017) with the definition as

$$
\left|\gamma_{1-2}(\theta)\right|=\frac{\left|\left\langle s_{1}(\theta) s_{2}^{*}(\theta)\right\rangle\right|}{\sqrt{\left\langle\left|s_{1}(\theta)\right|^{2}\right\rangle\left\langle\left|s_{2}(\theta)\right|^{2}\right\rangle}}
$$

Based on (3), the elements of $\mathbf{S}(\theta)$ are

$S_{H H}(\theta)=S_{H H} \cos ^{2} \theta+S_{H V} \cos \theta \sin \theta+S_{V H} \cos \theta \sin \theta+S_{V V} \sin ^{2} \theta$

$S_{H V}(\theta)=-S_{H H} \cos \theta \sin \theta+S_{H V} \cos ^{2} \theta-S_{V H} \sin ^{2} \theta+S_{V V} \cos \theta \sin \theta$

$S_{V H}(\theta)=-S_{H H} \cos \theta \sin \theta-S_{H V} \sin ^{2} \theta+S_{V H} \cos ^{2} \theta+S_{V V} \cos \theta \sin \theta$

$S_{V V}(\theta)=S_{H H} \sin ^{2} \theta-S_{H V} \cos \theta \sin \theta-S_{V H} \cos \theta \sin \theta+S_{V V} \cos ^{2} \theta$

and the four independent polarimetric coherence patterns are

$$
\begin{aligned}
& \left|\gamma_{H H-V V}(\theta)\right|=\frac{\left|\left\langle s_{H H}(\theta) s_{V V}^{*}(\theta)\right\rangle\right|}{\sqrt{\left\langle\left. s_{H H}(\theta)\right|^{2}\right\rangle\left\langle\left|s_{V V}(\theta)\right|^{2}\right\rangle}} \\
& \left|\gamma_{H H-H V}(\theta)\right|=\frac{\left|\left\langle s_{H H}(\theta) s_{H V}^{*}(\theta)\right\rangle\right|}{\sqrt{\left\langle\left|s_{H H}(\theta)\right|^{2}\right\rangle\left\langle\left|s_{H V}(\theta)\right|^{2}\right\rangle}} \\
& \left|\gamma_{(H H+V V)-H V}(\theta)\right|=\frac{\left|\left\langle s_{H H+V V}(\theta) s_{H V}^{*}(\theta)\right\rangle\right|}{\sqrt{\left\langle\left|s_{H H+V V}(\theta)\right|^{2}\right\rangle\left\langle\left|s_{H V}(\theta)\right|^{2}\right\rangle}}
\end{aligned}
$$

$\left|\gamma_{(H H-V V)-H V}(\theta)\right|=\frac{\left|\left\langle s_{H H-V V}(\theta) s_{H V}^{*}(\theta)\right\rangle\right|}{\sqrt{\left\langle\left|s_{H H-V V}(\theta)\right|^{2}\right\rangle\left\langle\left|s_{H V}(\theta)\right|^{2}\right\rangle}}$ 
Because the variation of aforementioned polarimetric coherence patterns in the rotation domain may contain rich hidden polarimetric information and provides the potential to understand and interpret the scattering properties of targets, a visualization and characterization tool of polarimetric coherence pattern is established and a set of hidden polarimetric features are proposed to quantitatively characterize each independent polarimetric coherence's variation along the radar line of sight (Chen et al. 2016b; Chen 2017). Using $|\gamma(\theta)|$ as an example, the definitions of these new polarimetric features are

1) Original Coherence $\gamma_{\text {-org }}$ :

$$
\gamma_{\text {-org }}=|\gamma(0)|
$$

2) Coherence Degree $\gamma_{\text {-mean }}$ :

$$
\gamma_{\text {-mean }}=\operatorname{mean}\{|\gamma(\theta)|\}
$$

3) Coherence Fluctuation $\gamma_{\text {-std }}$ :

$$
\gamma_{- \text {std }}=\operatorname{std}\{|\gamma(\theta)|\}
$$

4) Maximum Coherence $\gamma_{-\max }$ :

$$
\gamma_{-\max }=\max \{|\gamma(\theta)|\}
$$

5) Minimum Coherence $\gamma_{-\min }$ :

$$
\gamma_{-\min }=\min \{|\gamma(\theta)|\}
$$

6) Coherence Contrast $\gamma_{\text {-contrast }}$ :

$$
\gamma_{\text {-contrast }}=\gamma_{-\max }-\gamma_{-\min }
$$

7) Coherence Anisotropy $\gamma_{-A}$ :

$$
\gamma_{-\mathrm{A}}=\frac{\gamma_{-\max }-\gamma_{-\min }}{\gamma_{-\max }+\gamma_{-\min }}
$$

8) Coherence Beamwidth $\gamma_{-\mathrm{bw} \alpha}$ :

$$
\gamma_{-\mathrm{bw} \alpha}=\theta_{1}-\theta_{2}
$$

$$
\text { with }\left|\gamma\left(\theta_{1}\right)\right|=\left|\gamma\left(\theta_{2}\right)\right|=\alpha \times \gamma_{-\max } \text { and } \theta_{1}>\theta_{2}
$$

9) Maximum Rotation Angle $\theta_{\gamma-\max }$ :

$$
\theta_{\gamma-\max }=\underset{\theta \in[-\pi, \pi)}{\arg \max }\{|\gamma(\theta)|\}
$$

10) Minimum Rotation Angle $\theta_{\gamma-\min }$ :

$$
\theta_{\gamma-\min }=\underset{\theta \in[-\pi, \pi)}{\arg \min }\{|\gamma(\theta)|\}
$$

where mean $\{\cdot\}$ denotes the mean value, $\operatorname{std}\{\cdot\}$ denotes the standard deviation, $\max \{\cdot\}$ denotes the maximum value, $\min \{\cdot\}$ denotes the minimum value, $\alpha$ is the regulatory factor whose value is usually $0.95, \underset{\theta \in[-\pi, \pi)}{\arg \max }\{\cdot\}$ and $\underset{\theta \in[-\pi, \pi)}{\arg \min }\{\cdot\}$ denote the rotation angles within the main range $[-\pi, \pi)$ which produce $\gamma_{-\max }$ and $\gamma_{-\min }$ respectively.

From each independent polarimetric coherence of (22)-(25), aforementioned ten hidden polarimetric features (26)-(35) can be mined and extracted. Therefore, there are forty independent hidden polarimetric features derived from the visualization and characterization tool of polarimetric coherence pattern.

\section{CLASSIFICATION SCHEME BASED ON HIDDEN AND ROLL-INVARIANT POLARIMETRIC FEATURES}

Land cover classification is one of the important applications for understanding and interpreting PolSAR images. With the clear physical significances, the roll-invariant polarimetric features of $H / A n i / \bar{\alpha} /$ Span which relate to the targets' physical properties are commonly adopted in the classification schemes based on polarimetric features (Cloude and Pottier 1997). Because of the potential to discriminate different land covers of the hidden information mined in the rotation domain along the radar line of sight, this work aims at applying the selected new polarimetric features derived from the aforementioned two hidden polarimetric feature extraction methods to PolSAR land cover discrimination and classification investigation.

First of all, we select some suitable hidden polarimetric features according to the criterion of the class separation distance. Specifically, a certain number of land cover pairs are produced by combining each two land covers of the all. For each land cover pair, there is a hidden polarimetric feature which can maximize the class separation distance between the samples of the two land covers within it after normalization processing. Therefore, some hidden polarimetric features which are suitable for land cover discrimination are selected. Based on the AIRSAR and multi-temporal UAVSAR data described in detail in the subsequent section, the selected features of all the hidden polarimetric features derived in the rotation domain are $\theta_{0}-\operatorname{Re}\left[T_{12}(\theta)\right], \theta_{0}-\operatorname{Im}\left[T_{12}(\theta)\right], \theta_{0}-\operatorname{Re}\left[T_{23}(\theta)\right]$, $\gamma_{- \text {mean }}$ of $\left|\gamma_{(H H+V V)-H V}(\theta)\right|, \gamma_{- \text {org }}$ of $\left|\gamma_{(H H+V V)-H V}(\theta)\right|$, $\theta_{\gamma-\min }$ of $\left|\gamma_{(H H+V V)-H V}(\theta)\right|$ and $\gamma_{- \text {org }}$ of $\left|\gamma_{(H H-V V)-H V}(\theta)\right|$. Secondly, the combination of both the selected hidden polarimetric features and the roll-invariant polarimetric features of $H / A n i / \bar{\alpha} /$ Span is created to complement the land cover discrimination abilities of both. Finally, the support vector machine (SVM) with relatively good classification performance is chosen as the classifier, and the proposed classification scheme using the combination of the polarimetric features is illustrated in Figure 1. In order to extract the roll-invariant polarimetric features of $H / A n i / \bar{\alpha}$, the original PolSAR data must be speckle filtered. So we choose the recently reported adaptive SimiTest speckle filter (Chen et al. 2012).

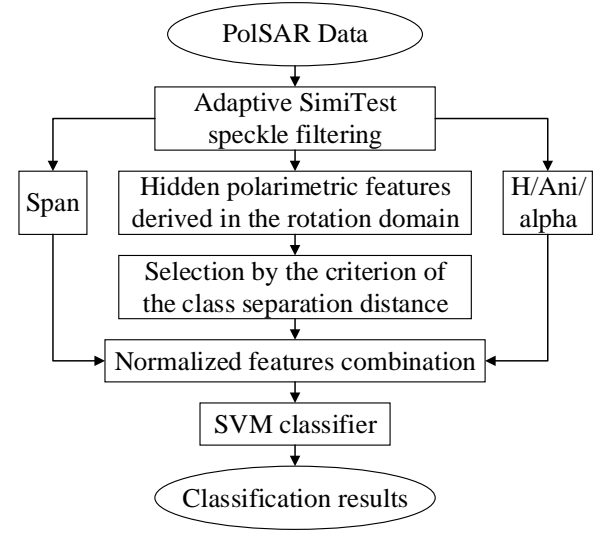

Figure 1 Flowchart of the proposed classification scheme

\section{COMPARISON EXPERIMENTS}

In order to demonstrate the added benefits from hidden polarimetric feature mining and selecting in the rotation domain and the advantage of combining the selected hidden polarimetric features with the roll-invariant polarimetric features of $H / A n i / \bar{\alpha} /$ Span to improve the classification accuracy, the proposed classification scheme is compared with the conventional one which only uses the roll-invariant polarimetric features of $H / A n i / \bar{\alpha} /$ Span. AIRSAR and multi-temporal UAVSAR data are adopted respectively in this 
section. They are both filtered by the adaptive SimiTest speckle filter with a $15 \times 15$ sliding window. All the pixels which have the corresponding ground-truth labels are used as the known samples for the training and validation processing. For each land cover in different PolSAR data respectively, a half of the known samples are randomly selected and used to train the SVM classifier, and the other half of the known samples are used for the followed validation. Besides, all the classification accuracies mentioned in this section are producer accuracies.

\subsection{Comparison with AIRSAR Data}

NASA/JPL AIRSAR L-band PolSAR data collected over Flevoland, the Netherlands, is adopted to compare the performance of the conventional and proposed classification schemes firstly. The range and azimuth pixel resolutions are $6.6 \mathrm{~m}$ and $12.1 \mathrm{~m}$ respectively. The RGB composite image of the filtered AIRSAR data with Pauli basis is shown in Figure 2(a). This study area contains various land covers and a ground-truth map for eleven known land covers (including stembeans, peas, forest, lucerne, wheat, beet, potatoes, bare soil, grasses, rapeseed and water) is shown in Figure 2(b).

With the conventional and proposed classification schemes, the classification results for the filtered AIRSAR data over the area with eleven known land covers are shown in Figure 3. And the classification accuracies of the eleven known land covers and overall are listed in Table 2. It is observed that the classification performance of the proposed classification scheme is better than that of the conventional one. The overall classification accuracy with the proposed classification scheme is $94.91 \%$, while that with the conventional classification scheme is $93.70 \%$. Moreover, for the most of these eleven land covers, the classification accuracies with the proposed classification scheme are higher than those with the conventional scheme. Especially for grasses, the classification accuracy increasing is $12.60 \%$. Finally, the classification results over the full-scene area with the conventional and proposed classification schemes respectively are shown in Figure 4.

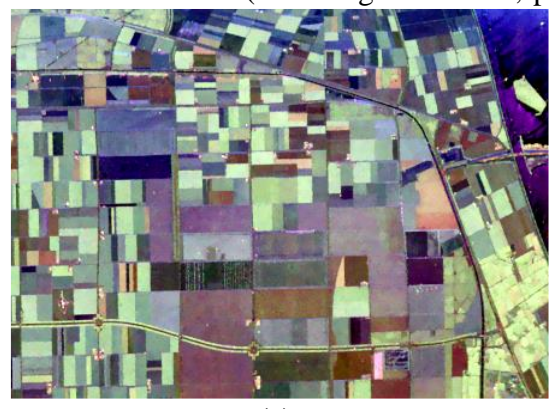

(a)

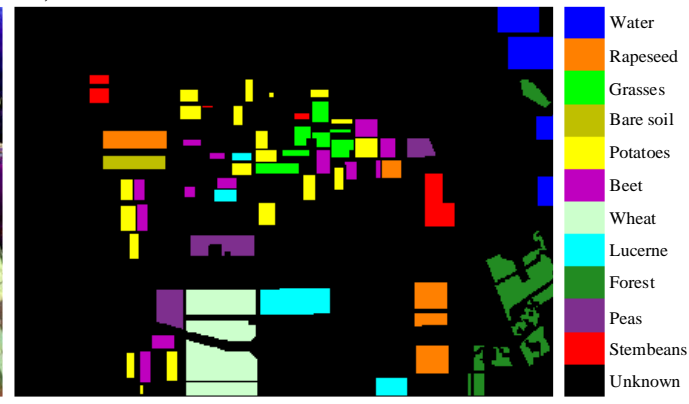

(b)

Figure 2 Study area. (a) The RGB composite image of the filtered AIRSAR data with Pauli basis,

(b) Ground-truth map

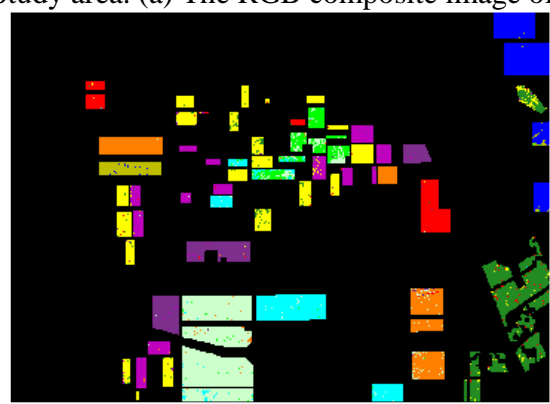

(a)

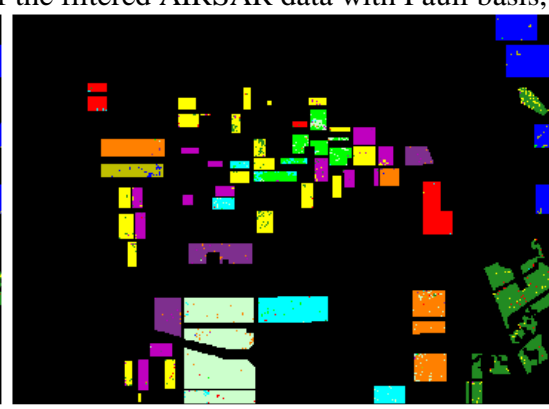

(b)

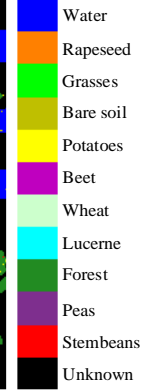

nknown

Figure 3 Classification results over the area with eleven known land covers. (a) and (b) are with the conventional and proposed classification schemes respectively

\begin{tabular}{|c|c|c|c|c|c|c|c|c|c|c|c|c|}
\hline & Stembeans & Peas & Forest & Lucerne & Wheat & Beet & Potatoes & $\begin{array}{c}\text { Bare } \\
\text { soil }\end{array}$ & Grasses & Rapeseed & Water & Overall \\
\hline $\begin{array}{l}\text { Conventional } \\
\text { scheme }\end{array}$ & 98.20 & 97.43 & 91.96 & 96.50 & 95.85 & 94.47 & 92.56 & 96.43 & 66.16 & 94.81 & 97.86 & 93.70 \\
\hline $\begin{array}{l}\text { Proposed } \\
\text { scheme }\end{array}$ & 98.30 & 96.82 & 93.85 & 96.64 & 97.21 & 95.86 & 92.58 & 91.36 & 78.76 & 95.79 & 98.08 & 94.91 \\
\hline
\end{tabular}

Table 2 Classification accuracies (\%) of the eleven known land covers and overall of the AIRSAR data

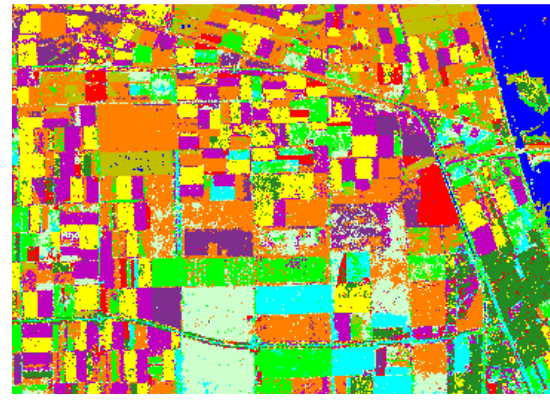

(a)

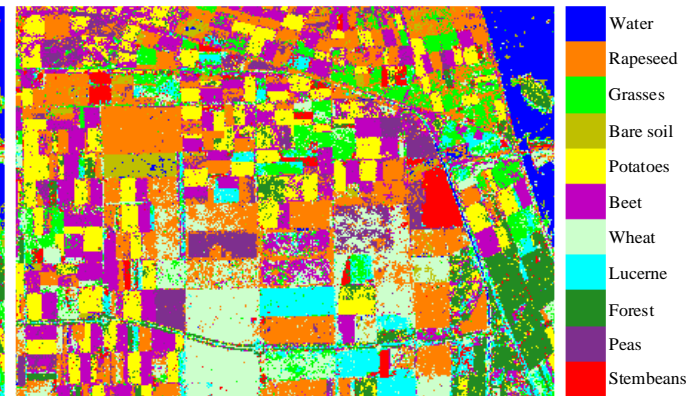

(b)

Figure 4 Classification results over the full-scene area. (a) and (b) are with the conventional and proposed classification schemes respectively 


\subsection{Comparison with Multi-Temporal UAVSAR Data}

Based on the results of the comparison experiment with AIRSAR data, NASA/JPL UAVSAR L-band PolSAR multitemporal data collected over Manitoba, Canada, are adopted to demonstrate the better classification performance of the proposed classification scheme further and its better robustness for multi-temporal PolSAR data. The range and azimuth pixel resolutions are $5 \mathrm{~m}$ and $7 \mathrm{~m}$ respectively. Four temporal data are used in this comparison. They are $17^{\text {th }}$ June, $22^{\text {th }}$ June, $3^{\text {rd }}$ July and $17^{\text {th }}$ July. The RGB composite images of the filtered multitemporal UAVSAR data with Pauli basis are shown in Figure 5. This study area contains various land covers and a ground-truth map for seven known land covers (including broadleaf, forage crops, soybeans, corn, wheat, rapeseed and oats) is shown in Figure 6.

With the conventional and proposed classification schemes, the classification results for the filtered multi-temporal UAVSAR data over the area with seven known land covers are shown in Figure 7. And the classification accuracies of the seven known land covers and overall are listed in Table 3 . It is very clear that the classification performance of the proposed

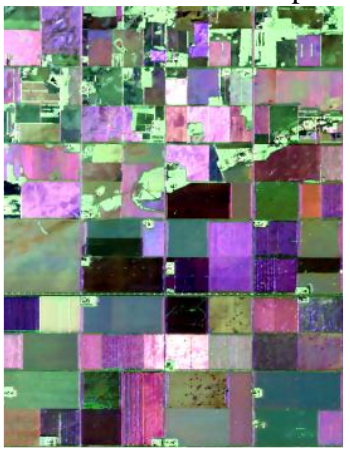

(a)

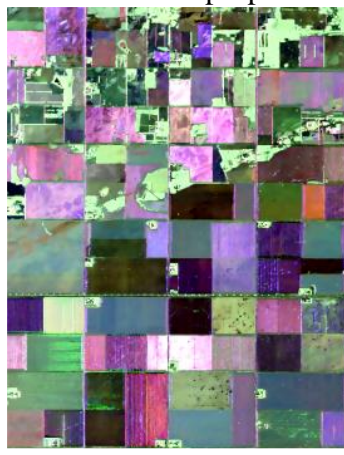

(b)

classification scheme is much better than that of the conventional one. The averaged overall classification accuracy with the proposed classification scheme for four temporal data is $97.08 \%$, which is much higher than the $87.79 \%$ from the conventional classification scheme. The overall classification accuracy increments for these four temporal $\left(17^{\text {th }} \mathrm{June}, 22^{\text {th }}\right.$ June, $3^{\text {rd }}$ July and $17^{\text {th }}$ July) data are $6.42 \%, 5.22 \%, 16.95 \%$ and $8.59 \%$ respectively. Moreover, the proposed classification scheme has better robustness for different temporal data. The better performance and higher accuracies of the proposed classification scheme are able to keep for the multi-temporal PolSAR data. Especially for forage crops, wheat and oats, the classification accuracy ranges for four temporal data with the conventional classification scheme are 54.42 64.34\%, $68.62 \sim 97.91 \%$ and $64.04 \sim 86.43 \%$, while those with the proposed classification scheme are 78.40 94.97\%, 96.91 98.29\% and 94.44 98.29\% respectively. Finally, the classification results over the full-scene area for these four temporal UAVSAR data with the conventional and proposed classification schemes are shown in Figure 8.

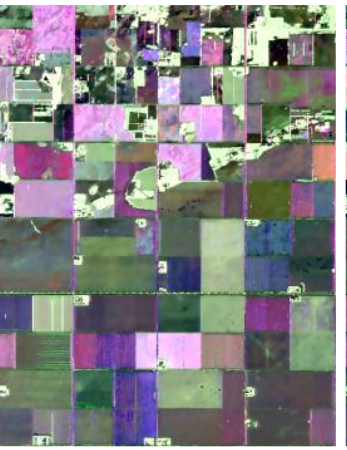

(c)

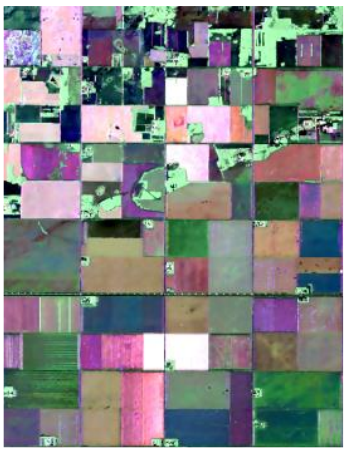

(d)

Figure 5 The RGB composite image of the filtered multi-temporal UAVSAR data with Pauli basis.

(a) $17^{\text {th }}$ June, (b) $22^{\text {th }}$ June, (c) $3^{\text {rd }}$ July, (d) $17^{\text {th }}$ July

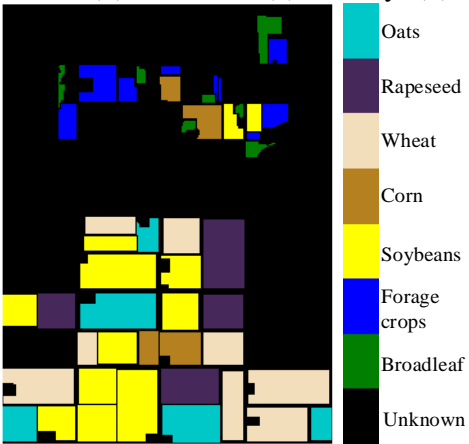

Figure 6 Ground-truth map for seven known land covers over the study area

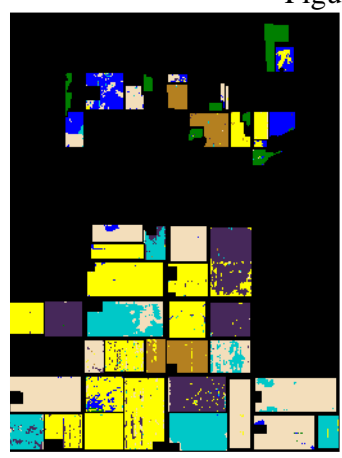

(a1)

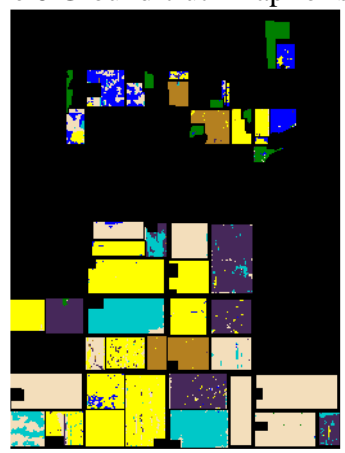

(b1)

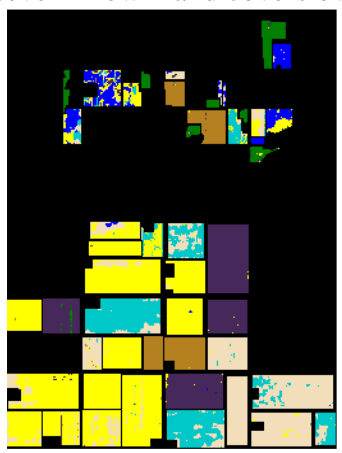

(c1)

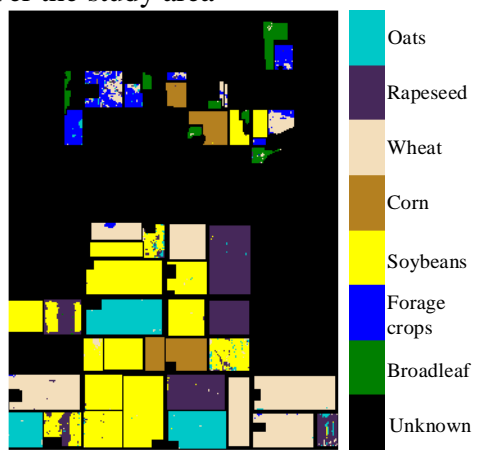

(d1) 

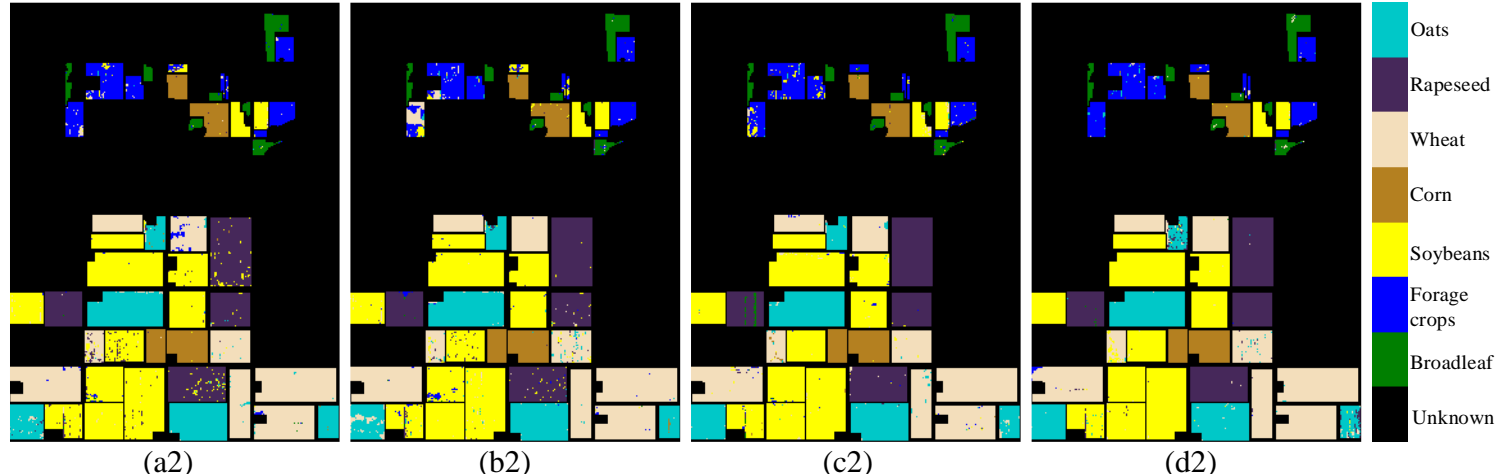

Figure 7 Classification results over the area with seven known land covers. (a1)-(d1) are $17^{\text {th }}$ June, $22^{\text {th }}$ June, $3^{\text {rd }}$ July and $17^{\text {th }}$ July with the conventional classification scheme, (a2)-(d2) are $17^{\text {th }}$ June, $22^{\text {th }}$ June, $3^{\text {rd }}$ July and $17^{\text {th }}$ July with the proposed classification scheme

\begin{tabular}{|c|c|c|c|c|c|c|c|c|c|}
\hline & $\begin{array}{c}\text { Classification } \\
\text { scheme }\end{array}$ & Broadleaf & $\begin{array}{c}\text { Forage } \\
\text { crops }\end{array}$ & Soybeans & Corn & Wheat & Rapeseed & Oats & Overall \\
\hline \multirow{2}{*}{$17^{\text {th }}$ June } & Conventional & $\mathbf{9 8 . 6 1}$ & 62.44 & 92.57 & 96.11 & 93.73 & 91.70 & 86.43 & 90.22 \\
\cline { 2 - 10 } & Proposed & 98.33 & $\mathbf{9 0 . 6 8}$ & $\mathbf{9 7 . 2 3}$ & $\mathbf{9 8 . 2 9}$ & $\mathbf{9 6 . 9 1}$ & $\mathbf{9 5 . 9 1}$ & $\mathbf{9 7 . 4 7}$ & $\mathbf{9 6 . 6 4}$ \\
\hline \multirow{2}{*}{$22^{\text {th }}$ June } & Conventional & $\mathbf{9 8 . 0 2}$ & 61.57 & 94.21 & 97.08 & $\mathbf{9 7 . 9 1}$ & 93.88 & 76.59 & 90.69 \\
\cline { 2 - 10 } & Proposed & 97.34 & $\mathbf{7 8 . 4 0}$ & $\mathbf{9 7 . 0 8}$ & $\mathbf{9 8 . 0 3}$ & 97.82 & $\mathbf{9 7 . 7 1}$ & $\mathbf{9 5 . 4 4}$ & $\mathbf{9 5 . 9 1}$ \\
\hline \multirow{2}{*}{$3^{\text {rd }}$ July } & Conventional & 97.07 & 54.42 & 90.19 & 98.79 & 68.62 & $\mathbf{9 8 . 7 6}$ & 64.04 & 80.82 \\
\cline { 2 - 10 } & Proposed & $\mathbf{9 7 . 8 0}$ & $\mathbf{8 9 . 6 9}$ & $\mathbf{9 8 . 3 1}$ & $\mathbf{9 9 . 0 1}$ & $\mathbf{9 8 . 2 9}$ & 98.48 & $\mathbf{9 8 . 2 9}$ & $\mathbf{9 7 . 7 7}$ \\
\hline \multirow{2}{*}{$17^{\text {th }}$ July } & Conventional & 96.59 & 64.34 & 97.26 & $\mathbf{9 9 . 7 3}$ & 84.90 & 92.32 & 83.22 & 89.41 \\
\cline { 2 - 10 } & Proposed & $\mathbf{9 6 . 8 5}$ & $\mathbf{9 4 . 9 7}$ & $\mathbf{9 9 . 4 0}$ & 99.65 & $\mathbf{9 7 . 8 1}$ & $\mathbf{9 9 . 7 2}$ & $\mathbf{9 4 . 4 4}$ & $\mathbf{9 8 . 0 0}$ \\
\hline \multirow{2}{*}{ Averaged } & Conventional & 97.57 & 60.69 & 93.56 & 97.93 & 86.29 & 94.17 & 77.57 & 87.79 \\
\cline { 2 - 10 } & Proposed & $\mathbf{9 7 . 5 8}$ & $\mathbf{8 8 . 4 4}$ & $\mathbf{9 8 . 0 1}$ & $\mathbf{9 8 . 7 5}$ & $\mathbf{9 7 . 7 1}$ & $\mathbf{9 7 . 9 6}$ & $\mathbf{9 6 . 4 1}$ & $\mathbf{9 7 . 0 8}$ \\
\hline
\end{tabular}

Table 3 Classification accuracies (\%) of the seven known land covers and overall of the multi-temporal UAVSAR data

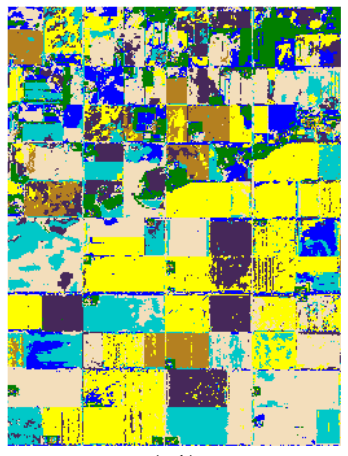

(a1)

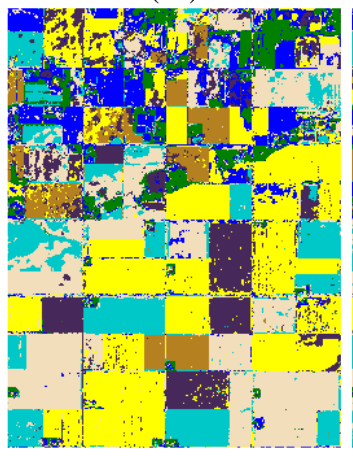

(a2)

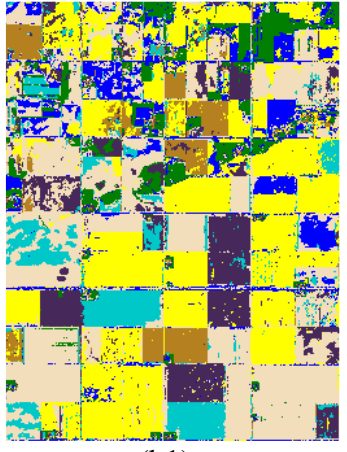

(b1)

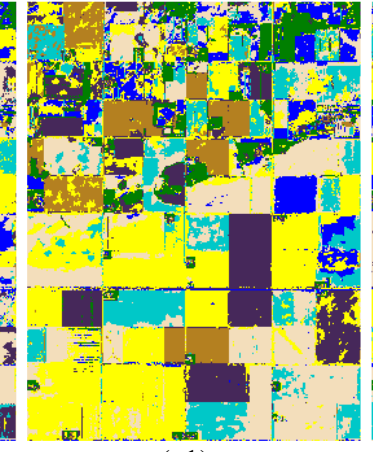

(c1)

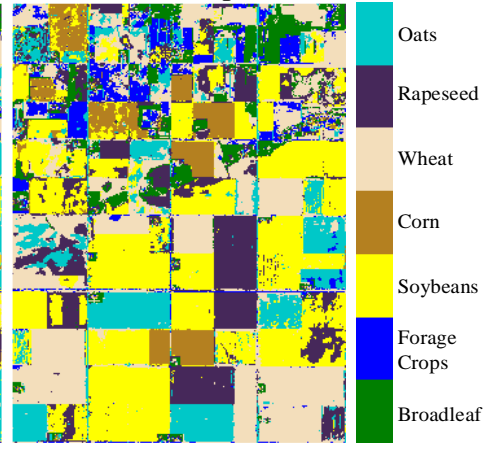

(d1)

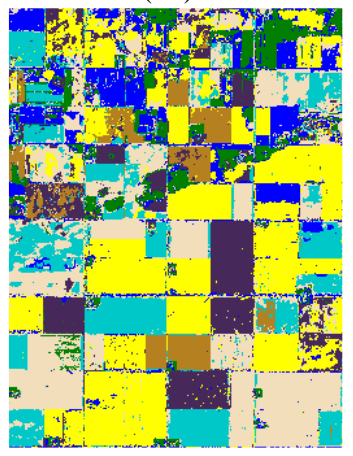

(b2)

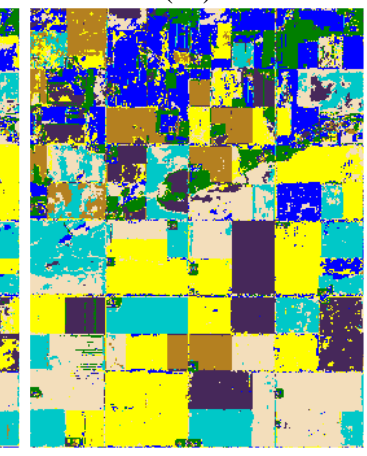

(c2)

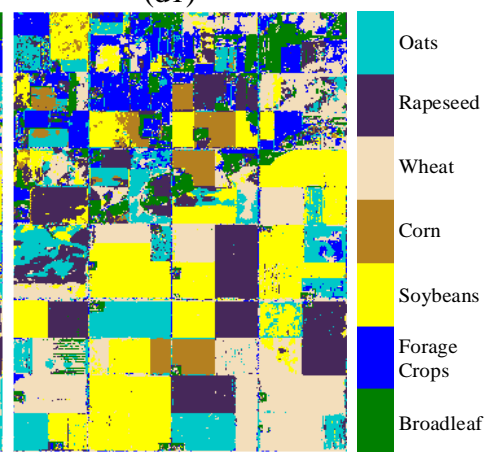

(d2)

Figure 8 Classification results over the full-scene area. (a1)-(d1) are $17^{\text {th }}$ June, $22^{\text {th }}$ June, $3^{\text {rd }}$ July and $17^{\text {th }}$ July with the conventional classification scheme, (a2)-(d2) are $17^{\text {th }}$ June, $22^{\text {th }}$ June, $3^{\text {rd }}$ July and $17^{\text {th }}$ July with the proposed classification scheme

CONCLUSIONS

The comparison experiments based on AIRSAR and multitemporal UAVSAR data respectively clearly demonstrate that mining and extracting hidden polarimetric features and information in the rotation domain along the radar line of sight can gain the added benefits in PolSAR land cover classification. With the added benefits, the land covers discrimination ability is enhanced and the classification accuracies of the most land covers and overall are improved significantly. Moreover, the classification scheme using the both selected hidden polarimetric features and roll-invariant polarimetric features can achieve better robustness for multi-temporal PolSAR data. This provides a new vision for PolSAR image interpretation and application. 


\section{ACKNOWLEDGEMENTS}

This work was supported in part by the National Natural Science Foundation of China under Grants 41301490, 61490690 and 61490692.

\section{REFERENCES}

Cloude, S. R., and E. Pottier, 1996. A review of target decomposition theorems in radar polarimetry. IEEE Transactions on Geoscience and Remote Sensing, 34(2), pp. 498-518.

Cloude, S. R., and E. Pottier, 1997. An entropy based classification scheme for land applications of polarimetric SARs. IEEE Transactions on Geoscience and Remote Sensing, 35(1), pp. 68-78.

Chen, S.-W., X.-S. Wang, and M. Sato, 2012. PolInSAR complex coherence estimation based on covariance matrix similarity test. IEEE Transactions on Geoscience and Remote Sensing, 50(11), pp. 4699-4709.

Chen, S.-W., and M. Sato, 2013. Tsunami damage investigation of built-up areas using multitemporal spaceborne full polarimetric SAR images. IEEE Transactions on Geoscience and Remote Sensing, 51(4), pp. 1985-1997.

Chen, S.-W., X.-S. Wang, S.-P. Xiao, and M. Sato, 2014a. General polarimetric model-based decomposition for coherency matrix. IEEE Transactions on Geoscience and Remote Sensing, 52(3), pp. 1843-1855.

Chen, S.-W., Y.-Z. Li, X.-S. Wang, S.-P. Xiao, and M. Sato, 2014b. Modeling and interpretation of scattering mechanisms in polarimetric synthetic aperture radar: Advances and perspectives. IEEE Signal Processing Magazine, 31(4), pp. 79 89.

Chen, S.-W., X.-S. Wang, and M. Sato, 2014c. Uniform polarimetric matrix rotation theory and its applications. IEEE Transactions on Geoscience and Remote Sensing, 52(8), pp. 4756-4770.

Chen, S.-W., Y.-Z. Li, and X.-S. Wang, 2015. Crop discrimination based on polarimetric correlation coefficients optimization for PolSAR data. International Journal of Remote Sensing, 36(16), pp. 4233-4249.

Chen, S.-W., X.-S. Wang, and M. Sato, 2016a. Urban damage level mapping based on scattering mechanism investigation using fully polarimetric SAR data for the 3.11 East Japan earthquake. IEEE Transactions on Geoscience and Remote Sensing, 54(12), pp. 6919-6929.

Chen, S.-W., Y.-Z. Li, and X.-S. Wang, 2016b. A visualization tool for polarimetric SAR data investigation. The 11th European Synthetic Aperture Radar Conference, Hamburg, Germany, pp. 579-582.

Chen, S.-W., 2017. Polarimetric coherence pattern: A visualization and characterization tool for PolSAR data investigation. IEEE Transactions on Geoscience and Remote Sensing, Under Review.
Chang, C.-C., and C.-J. Lin, 2011. LIBSVM: A library for support vector machines. ACM Transactions on Intelligent Systems and Technology, 2(3), pp. 389-396.

Freeman, A., and S. L. Durden, 1998. A three-component scattering model for polarimetric SAR data. IEEE Transactions on Geoscience and Remote Sensing, 36(3), pp. 963-973.

Lee, J.-S., M. R. Grunes, T. L. Ainsworth, L. Du, D. L. Schuler, and S. R. Cloude, 1999. Unsupervised classification using polarimetric decomposition and the complex Wishart classifier. IEEE Transactions on Geoscience and Remote Sensing, 37(5), pp. $2249-2258$

Lee, J.-S., and E. Pottier, 2009. Polarimetric Radar Imaging: From Basics to Applications. CRC, Press.

Wu, Y.-H., K.-F. Ji, and W.-X. Yu, 2007. A new feature selection algorithm for SVM-based fully polarimetric SAR image classification. Signal Processing, 23(6), pp. 877-881.

Xiao, S.-P., S.-W. Chen, Y.-L. Chang, Y.-Z. Li, and M. Sato, 2014. Polarimetric coherence optimization and its application for manmade target extraction in PolSAR data. IEICE Transactions on Electronics, E97C(6), pp. 566-574.

Yamaguchi, Y., T. Moriyama, M. Ishido, and H. Yamada, 2005. Four-component scattering model for polarimetric SAR image decomposition. IEEE Transactions on Geoscience and Remote Sensing, 43(8), pp. 1699-1706.

Zhou, Y., H.-P. Wang, F. Xu, and Y.-Q. Jin, 2016. Polarimetric SAR images classification using deep convolutional neural networks. IEEE Geoscience and Remote Sensing Letters, 13(12), pp. 1935-1939. 